OPEN ACCESS

Edited by:

Angela Fang,

University of Washington,

United States

Reviewed by:

Warren Mansell,

The University of Manchester,

United Kingdom

Sarita Sood,

University of Jammu, India

*Correspondence:

Preethi Premkumar

premkump@/sbu.ac.uk

Specialty section: This article was submitted to

Psychological Therapies,

a section of the journal

Frontiers in Psychiatry

Received: 13 April 2021

Accepted: 30 June 2021

Published: 19 August 2021

Citation:

Premkumar P, Heym N, Brown DJ, Battersby S, Sumich A, Huntington B,

Daly $R$ and Zysk E (2021) The

Effectiveness of Self-Guided Virtual-Reality Exposure Therapy for

Public-Speaking Anxiety.

Front. Psychiatry 12:694610

doi: 10.3389/fpsyt.2021.694610

\section{The Effectiveness of Self-Guided Virtual-Reality Exposure Therapy for Public-Speaking Anxiety}

\author{
Preethi Premkumar ${ }^{1 *}$, Nadja Heym ${ }^{2}$, David Joseph Brown ${ }^{3}$, Steven Battersby ${ }^{3}$, \\ Alexander Sumich ${ }^{2}$, Bethany Huntington ${ }^{4}$, Rosie Daly ${ }^{2}$ and Eva Zysk ${ }^{5}$
}

${ }^{1}$ Division of Psychology, London South Bank University, London, United Kingdom, ${ }^{2}$ Department of Psychology, Nottingham Trent University, Nottingham, United Kingdom, ${ }^{3}$ Department of Computer Science, Nottingham Trent University, Nottingham, United Kingdom, ${ }^{4}$ Department of Psychology, University of Nottingham, Nottingham, United Kingdom, ${ }^{5}$ Department of

Psychology, University of British Columbia, Vancouver, BC, Canada

Objectives: Self-guided virtual-reality exposure therapy (VRET) is a psychological intervention that enables a person to increase their own exposure to perceived threat. Public-speaking anxiety (PSA) is an anxiety-provoking social situation that is characterized by fear of negative evaluation from an audience. This pilot study aimed to determine whether self-guided VRET (1) increases exposure to PSA-specific virtual social threats, and (2) reduces anxiety, arousal, heartrate and PSA over repeated exposure.

Methods: Thirty-two University students (27 completers) with high self-reported public-speaking anxiety attended 2 weekly self-guided VRET sessions. Each session involved the participant delivering a 20-min speech in a virtual classroom. Participants were able to increase their exposure to virtual social threat through the audience size, audience reaction, number of speech prompts, and their own salience in the virtual classroom at 4-min intervals. Participants' heartrates and self-reported anxiety and arousal were monitored during these intervals. Participants completed psychometric assessments after each session and 1 month later.

Results: Participants increased their exposure to virtual social threat during each VRET session, which coincided with a reduction in heartrate and self-reported anxiety and arousal. Improvement in PSA occurred post-treatment and 1 month later. The in-session improvement in anxiety correlated with reductions in fear of negative evaluation post-treatment and 1 month later.

Conclusions: Increased self-exposure to virtual social threat from self-guided VRET relieves anxiety and shows immediate reductions in subjective and physiological arousal during application, but also yields sustained improvement in PSA.

Keywords: virtual audience, heart rate, negative evaluation, perceived control, social anxiety, head-mounted display

\section{INTRODUCTION}

Social anxiety is, in part, an exaggerated fear of being negatively evaluated by others, for example being criticized, humiliated or rejected during social interaction, observation, and/or in performance situations (1). People with social anxiety disorder (SAD) may appear shy and withdrawn in social situations to mask their immense discomfort and may sometimes avoid 
social situations altogether (1). SAD has a lifetime prevalence of $4 \%$ as per a large multinational epidemiological survey (2). SAD is said to be the third most common psychiatric disorder (3). SAD affects personal relationships, work engagement and academic achievement $(4,5)$. Yet, SAD is often underdiagnosed (6) and undertreated, with over $80 \%$ of people diagnosed with SAD not seeking treatment or having typically lived with their symptoms for 15 to 20 years before seeking treatment (7). Individuals with SAD may not seek treatment for reasons, such as avoidance of face-to-face contact, lack of confidence in treatment, and financial costs $(8,9)$. Thus, SAD being both highly prevalent and under-treated makes it a large public health concern with psychological and economic costs to the individual and society.

Cognitive-behavioral therapy (CBT), which includes exposure therapy, has become the most evidenced form of intervention for SAD $(10,11)$. The cognitive element of CBT encourages the patient to question their maladaptive beliefs (10). The exposure element gradually increases the patient's exposure to real (in vivo) or imagined social threat. CBT works in a group format and is a natural setting to test social fears with group members (12). Over the last two decades, virtual-reality exposure therapy (VRET) has become a popular digital intervention for various psychological disorders $(13,14)$. A systematic review of 10 studies showed that VRET was as effective as in vivo exposure therapy post-intervention (15). Moreover, a meta-analysis found a large effect size favoring VRET for SAD over waitlist, but a small effect size favoring in vivo (i.e., face-to-face) exposure therapy with a therapist over VRET based on six studies (13). In vivo exposure therapy may appear to favor VRET for SAD partly because in vivo exposure therapy offers a wider range of social situations to rehearse exposure (16). While in vivo exposure is effective, many people with social anxiety refuse treatment due to their fear of social situations and the very nature of therapy being a social situation.

VRET is a viable alternative to in vivo exposure therapy because patients can encounter social threat in a safe and more predictable virtual environment, and feel that they have control over their exposure to their perceived threat (17). VRET could engage treatment refusers and it has shown efficacy among those who undergo it. VRET may be effective because it could address cognitive biases associated with real social threats, such as having fearful thoughts during public speaking (18) and emotional problems, such as avoidance of and hyperarousal from threat (17). Taken together, VRET offers a promising solution to reduce overall rates of SAD in the population.

Public-speaking anxiety (PSA) is a variant of social anxiety that is frequently encountered by students (19). PSA is a highly anxiety-provoking social situation; it impairs up to $97 \%$ of socially anxious individuals (20) and affects $77 \%$ of the general population (21). Delivering a public speech in a virtual environment induces as much distress and physiological arousal as delivering a public speech in front of a live audience (22). It significantly increases anxiety and heartrate in socially anxious individuals $(22,23)$. Research has confirmed that virtual exposure translates to "real life" threat, such as PSA (22). Exposure therapy for social threat often entails delivering a public speech in front of a real or virtual audience $(24,25)$. VRET can systematically manipulate these social threats, which can induce strong cognitions and high intensity levels of fear $(26,27)$. These VRET-led improvements in social anxiety are long-lasting and generalize to real world situations (28).

\section{Self-Guided vs. Therapist-Led VRET for SAD}

Therapist-led VRET is where the therapist controls the level of graded virtual exposure according to the patient's hierarchy of fears $(16,24)$. Self-guided VRET is where the patient controls their own gradual exposure to virtual threat [e.g., (29)]. Selfguided VRET is seen as the latest advance in VRET technology and it produces a meaningful improvement (30). A benefit of self-guided VRET is that it can be easily delivered as homework alongside therapist-led sessions (9). Eight sessions of self-guided VRET for SAD were delivered to individuals with SAD and health controls. These sessions consisted of publicspeaking and they produced greater improvement in social anxiety among individuals with SAD than healthy controls (9). Even a single session of self-guided VRET for SAD produced a large improvement in PSA in individuals selfreporting high PSA (31). Two studies on acrophobia (fear of heights) found that symptoms of acrophobia improved to a greater extent (with large effect sizes) when receiving six modules of VR-CBT from a virtual therapist over 2 or 3 weeks compared to the wait-list group $(32,33)$. One likely reason for the efficacy of self-guided VRET is perceived control. According to the Health Belief Model, patients are more likely to engage in and comply with therapy if they believe to have control over treatment $(34,35)$. Such perceived control could denote resilience to social stress (36), decisionmaking (37) and cognitive reappraisal (38). Therapist-led VRET focuses more on establishing a good therapeutic alliance through agreement on therapeutic tasks and goals to achieve visible treatment outcomes, such as treatment adherence (39). Therapist-led VRET focuses less on supporting the client toward gaining autonomy (39) and control over exposure without risk of over-exposure to threat (40). Having a higher level of perceived control over exposure to threat encourages the person to approach the threat, at least among individuals with arachnophobia (40).

Self-guided VRET could enable such autonomy and control. According to the perceptual control theory (41), control involves keeping a perceptual variable (e.g., perceived distance from a threat) at a selected state through comparing its current value with a reference value that drives actions to counteract disturbances to that variable. 'Perceived' control could be defined as the consciously reportable experience of the amount of control over a specific variable (e.g., the verbal report of amount of control over perceived distance from a threat). Self-guided VRET could enhance control through providing a hierarchy of virtual threats and allowing the client to select the steps needed to reach a goal through graded exposure, for example, gradually reducing the distance from the audience. Future studies of self-guided VRET should assess client control within the virtual environment and how it affects the effectiveness of the intervention. 
Manipulating certain elements of virtual social threat during self-guided exposure could improve the efficacy of self-guided VRET $(42,43)$. These social elements could include (1) the audience size (24), (2) the reaction of the avatar audience (26), (3) the proximity to the audience (31), (4) the number of speech prompts available for delivering a speech (44), and (5) the salience or presence of the self in the virtual classroom (45). Manipulating the audience size is well-documented to increase exposure within VRET for SAD [c.f. (24)]. In contrast, the reaction of the audience has been manipulated less often, with studies often defaulting to a neutral audience reaction (44). Manipulating the audience reaction is crucial for addressing the fear of human evaluation, whether positive or negative, as the fear of human evaluation is a core fear of social anxiety (46, 47). Fear of negative evaluation predicts response to treatment for SAD (48). Importantly, negative reactions from the virtual audience have been found to evoke social anxiety in spite of participants being aware that the members of the audience are merely fictitious (26). The proximity to the audience is another factor to be considered for manipulation, as this manipulation could alter the attention of the participants to the audience. Being closer to the audience could encourage the socially anxious person to focus on the audience rather than themselves, thus improving eye-contact and fluency (49). The speaker's close proximity to the audience, especially among individuals with PSA, could mimic the feeling of their performance being closely scrutinized (50). Thus, gradual exposure could help to overcome this sense of scrutiny. Salience of the self in the virtual classroom is another factor that could gauge the speaker's awareness of being in the virtual space and increase their sense of presence. Creating a sense of presence in the virtual environment is important. Presence is the participant's psychological response to a virtual environment (45) in terms of their sense of immersion and emotions, such as anxiety $(51,52)$. A head-mounted display of virtual social interactions increases presence more than a screen-projected display does (53).

Measuring physiological arousal during VRET would objectively measure speech and performance anxiety. Delivering a speech in front of a virtual audience increases anxiety and heartrate in socially anxious individuals $(22,23)$. In contrast, patients with SAD have a lower heartrate than people with moderate social anxiety while monitoring their own performance when under public scrutiny (54); this finding could suggest a breakdown of the physiological stress response system due to performance anxiety. The physiological stress response is compromised in clinical social anxiety; yet, a 4-week therapist-guided VRET for PSA reduces heartrate (55). Thus, physiological arousal could be an objective measure of the psychological response to VRET.

The current pilot study aimed to test the feasibility of selfguided VRET for PSA in a sub-clinical group of university students who self-reported high PSA. It was hypothesized that (1) participants would gradually increase their exposure at their own pace to the five aforementioned elements of social threat during the self-guided VRET; (2) the gradual exposure to social threat would produce a concomitant reduction in anxiety, arousal and heartrate within the virtual environment; (3) self-guided VRET would reduce PSA at post-intervention and 1-month follow-up timepoints, and (4) changes in anxiety, arousal and heartrate during the VRET sessions would relate to improvement in PSA at post-intervention and 1-month follow-up timepoints.

\section{MATERIALS AND METHODS}

\section{Participants}

Thirty-two participants were invited to take part in the experiment on the basis of scoring the highest on the Speech Anxiety Thoughts Inventory (SATI) (56) among a large participant pool of 336 students. These 336 students were recruited for potential inclusion in this social anxiety study if they met the inclusion criteria and had completed the SATI in an online survey among other several self-report measures (see section Materials and Assessments). The 32 participants greatly surpassed the inclusion criterion of scoring 1.5 SD above the mean SATI score $[$ mean $(S D)=54.34(18.35)]$ in an independent normative sample $(n=548)$ (56). The mean (SD, range) SATI score $=96.7(7.8,82-111)$ of the 32 participants was $2.3 \mathrm{SD}$ above the mean of the normative sample (56) and one SD above the mean of the current screening survey sample $(n=$ 336). Further inclusion criteria were being aged $18+$ years, a university student, able to speak English fluently and having normal or corrected vision with contact lenses. Participants' ages ranged from 18 to 40 years (mean $=21.4, \mathrm{SD}=4.9$ ) and mostly identified as female $(n=27,84.4 \%)$ (see Table 1). All participants were psychology students (28 undergraduates, 4 postgraduates). Twenty-seven $(84.4 \%)$ were Caucasian, three were African-Caribbean, one was Asian and one was mixed race. English was either their first language (87.5\%) or second language (12.5\%). Participants ranged from never having been diagnosed with SAD $(84 \%)$ to having a current diagnosis $(6.0 \%)$ or a past diagnosis of SAD $(6.0 \%)$; one participant chose not to declare their diagnostic status. Individuals who were currently engaging in SAD psychotherapy were excluded.

\section{Assessments}

\section{Speech Anxiety Thoughts Inventory}

This 23-item scale assesses negative thoughts related to speech anxiety, such as "I worry that I will be asked to give a speech." Items are rated from 1 ("I do not believe the statement at all") to 5 ("I completely believe the statement"). The overall score was the total of individual items. The scale has two factors, namely 'prediction of poor performance' and 'fear of negative evaluation by audience'. The mean (SD) of the SATI has been previously reported to be $54.34(\mathrm{SD}=18.35)$ in Psychology undergraduate students $(n=547)$ (56). The scale has convergent validity with other measures of public speaking (56). Internal consistency was good in the current sample (Cronbach's $\alpha=0.80$ ).

\section{Public Speaking Anxiety Scale}

The PSAS assesses the manifestation of cognitive, behavioral and physiological responses to PSA. It contains 17 items, such as "Giving a speech is terrifying." Each item is scored from 1 ("Not at all") to 5 ("Extremely"), with 5 items being reverse-coded. The mean score of individual items is calculated. The scale has 
TABLE 1 | Demographic characteristics and social anxiety of completers $(n=21)$ and non-completers $(n=11)$.

\begin{tabular}{|c|c|c|c|c|c|}
\hline Characteristic & Completers & Non-completers & $\begin{array}{c}\text { T statistic or } \\
\text { chi-square }(d f)\end{array}$ & $P$-value & Effect size $\left(\eta^{2}\right)$ \\
\hline$N$ & 21 & 11 & & & \\
\hline Age, mean (S.D.) & $21.57(5.00)$ & $21.00(4.98)$ & $0.3(30)$ & 0.760 & 0.11 \\
\hline Gender, \% female & 76.2 & 100 & 3.1 & 0.08 & \\
\hline Ethnicity, \% White & 90.5 & 72.5 & 1.7 & 0.19 & \\
\hline SAD diagnosis, \% & 9.5 & 18.2 & 0.5 & 0.48 & \\
\hline \multicolumn{6}{|c|}{ Social anxiety at baseline } \\
\hline SATI & $97.71(7.44)$ & $94.64(8.35)$ & $1.07(30)$ & 0.295 & 0.40 \\
\hline PSAS $^{*}$ & $4.26(0.31)$ & $4.3(0.66)$ & $0.39(30)$ & 0.693 & 0.15 \\
\hline $\mathrm{PRCS}^{*}$ & $1.17(0.10)$ & $1.19(0.20)$ & $0.43(30)$ & 0.669 & 0.16 \\
\hline $\mathrm{BFNE}^{\star}$ & $50.67(7.14)$ & $47.81(13.62)$ & $0.78(30)$ & 0.440 & 0.29 \\
\hline LSPS-P-anx & $20.29(6.10)$ & $21.87(6.9)$ & $0.65(30)$ & 0.523 & 0.24 \\
\hline LSPS-P-avoid & $18.62(6.14)$ & $16.54(7.53)$ & $0.84(30)$ & 0.408 & 0.31 \\
\hline LSPS-S-anx & $18.57(8.18)$ & $18.91(6.95)$ & $0.12(30)$ & 0.908 & 0.04 \\
\hline LSPS-S-avoid & $17.00(7.79)$ & $15.91(7.27)$ & $0.38(30)$ & 0.703 & 0.14 \\
\hline SUDS avoidance & 85.71 (22.26) & $97.27(2.47)$ & $1.67(30)$ & 0.105 & 0.62 \\
\hline \multicolumn{6}{|c|}{ Social anxiety at session one } \\
\hline $\mathrm{SATI}^{*}$ & $87.86(15.05)$ & $79.00(24.71)$ & $1.26(30)$ & 0.290 & 0.47 \\
\hline PSA & $3.87(0.59)$ & $3.57(0.75)$ & $1.22(30)$ & 0.234 & 0.45 \\
\hline PRCS-SF & $1.25(0.16)$ & $1.32(0.28)$ & $0.82(30)$ & 0.418 & 0.31 \\
\hline SUDS avoidance & 56.67 (20.33) & $53.64(25.80)$ & $0.36(30)$ & 0.718 & 0.14 \\
\hline
\end{tabular}

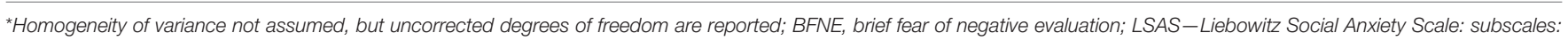

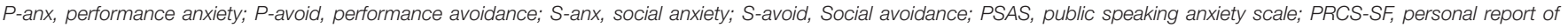
confidence as a speaker-short form; SATI, social anxiety thoughts inventory.

demonstrated concurrent, convergent and discriminant validity, and high internal consistency (Cronbach's $\alpha=0.94$ ) in a previous study (57), and good internal consistency (Cronbach's $\alpha=0.85$ ) in the current study.

\section{Personal Report of Confidence as a Speaker-Short Form}

The PRCS-SF is a 12-item scale that assesses behavioral responses, such as "My posture feels strained and unnatural." It assesses affective responses to public-speaking situations, such as "I am fearful and tense all the while I am speaking before a group of people." Participants answer "True" $=1$ or "False" $=2$ for each item. The overall score was calculated as the mean of individual items, so that the overall score ranged from 1 to 2 , with a higher score indicating more confidence as a speaker. The PRCS-SF had good internal consistency in a previous study (Cronbach's $\alpha=$ 0.85 ) (58), but was weaker in the current study (Cronbach's $\alpha=$ $0.60)$. The PRCS-SF has good convergent validity as determined by its relationship with measures of public-speaking ability (59).

\section{Liebowitz Social Anxiety Scale}

This 24-item scale (60) assesses fear and avoidance of social interaction situations, such as attending a party and meeting strangers, and performance situations, such as eating in public and taking a test. Each situation is assessed from 0 (none) to 4 (severe) on fear, and from 0 (never: $0 \%$ ) to 3 (usually: 67-100\%) on frequency of avoidance. The overall score and subscale scores are the totals of individual items. The scale has four subscales with the following means (SD) in a normative sample of 382 patients with SAD (61): Fear of Social Interaction = 16.9 (7.7); Avoidance of Social Interaction $=15.7$ (8.2); Fear of Performance $=18.6$ (6.8); and Avoidance of Performance $=16.0$ (7.3). The scale has shown convergent validity with other measures of social phobia and good internal consistency (Cronbach's $\alpha=0.96$ ) in a previous study (61) and the current study, $\alpha=0.96$.

\section{Brief Fear of Negative Evaluation Revised Scale}

This 12-item measure of fear of negative evaluation includes items, such as "I am frequently afraid of other people noticing my shortcomings." Items are rated from 0 ("Not at all characteristic of me") to 4 ("Extremely characteristic of me"). The overall score is the total score of individual items after reverse-coding positively-worded items. The mean (SD) of the BFNE in a sample of 201 undergraduate students was 30.7 (9.04) (62). The scale has shown discriminant and convergent validity and good internal consistency (Cronbach's $\alpha=0.97$ ) in a previous study (62) and the current sample.

\section{Subjective Units of Distress Scale}

The SUDS (63) is a visual analog scale that reliably measures subjective fear (64). It is sensitive to change in mental state (65). The SUDSs for anxiety and arousal were integrated and administered directly in the virtual environment through a scale ranging from "Not at all" (0) to "Extremely" (100). The anxiety and arousal questions were "How anxious do you feel right now?" and "How aroused do you feel right now?" Anxiety was 


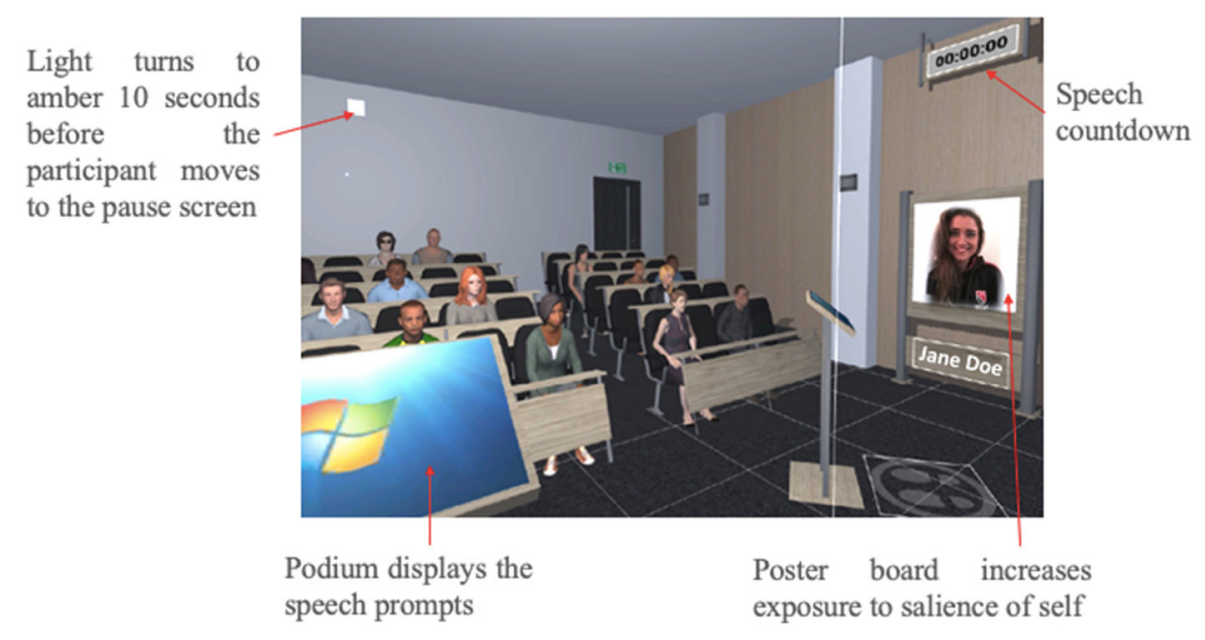

FIGURE 1 | Display of the features of the virtual classroom.

defined as dryness of mouth, difficulty breathing, trembling, feeling panicked and increased heart rate (66). Arousal was defined as feeling active, vigorous, lively, energetic and alert, and not tired, sleepy, drowsy, or passive (67). The behavioral avoidance question was "How much do you wish to avoid giving another speech?", and it was administered before and after each VR session along with the other self-report scales, where participants responded on a 0-10 Likert scale from "Not at all" to "Very much."

\section{Heart Rate}

Heartrate was measured from Microsoft Band 2, a biometric wristband, during the four 1-min intervals following each speech block over the 20-min VRET-led speech. Heartrate was sampled every $4 \mathrm{~s}$. The average heartrate was calculated in beats per minute during each of the four intervals.

\section{Virtual-Reality Exposure Therapy Software and Hardware}

The VRET was developed using the Unity real-time 3D development platform (68). The Unity-based VRET smartphone application was deployed to the Android operating system. Data on heartrate were collected through the smartphone application, since the VRET smartphone application was connected to the Microsoft Band 2, a biometric wristband. A bespoke plugin developed in Java acted as a bridge between the Java-based official Microsoft Band software development kit and the VRET smartphone application. A Samsung Gear VR headset housed a Samsung Galaxy S7 smartphone on which the VRET application ran to display the virtual environment.

\section{Virtual Environment Design and Self-Guided Manipulation}

Participants gave a 20 -min speech in a virtual classroom on the topic of "the experience of being a university student" following a previous study (45). The speech was broken into four 5-min blocks. Participants spoke spontaneously by following prompts that appeared in the virtual environment. The prompts included general knowledge about the University and its facilities, impressions about the course, level of academic support, extracurricular activities and social activities. Participants were encouraged to increase their exposure to the virtual social threat at their own pace. After every 5-min speech block, participants had a brief ( 1 minute) interval when they entered a virtual pause menu. Here, participants could respond to the SUDS on anxiety and arousal and navigate to a settings menu where they could manipulate the five elements of social threat (Figure 1). Each modifiable element had three grades $(G)$ of exposure, from low, moderate to high level of exposure: (i) audience sizesix (G1), 12 (G2) or 20 (G3) people; (ii) audience reactionapproving (G1), neutral (G2) or disapproving (G3); (iii) speaker's distance from the audience-far (G1), near (G2) or nearest (G3); (iv) number of speech prompts per slide-many (G1), moderate (G2) or few (G3); and (v) salience of self-no poster (G1), a silhouette with the label "Speaker" (G2), or a photo of the participant and their full name (G3). The speech prompts (with suggested points to speak about) appeared on the virtual podium as bullet points on PowerPoint slides through which the participant could scroll using the controls on the Samsung Gear VR headset. All participants were started on Grade 1 of each element of the VRET settings at Session 1. A countdown appeared inside the virtual classroom to allow participants to track the remaining time of their speech. Participants were given a 10-s warning by means of a signal turning from white to amber in the virtual lecture room before they were taken to the pause menu.

Due to a programming error, the podium disappeared when the participants changed their position from the default position to a different position; however, most participants chose not to manipulate the distance from the audience. Hence, the analyses excluded the data on the manipulation of distance from audience. 
Assessments completed

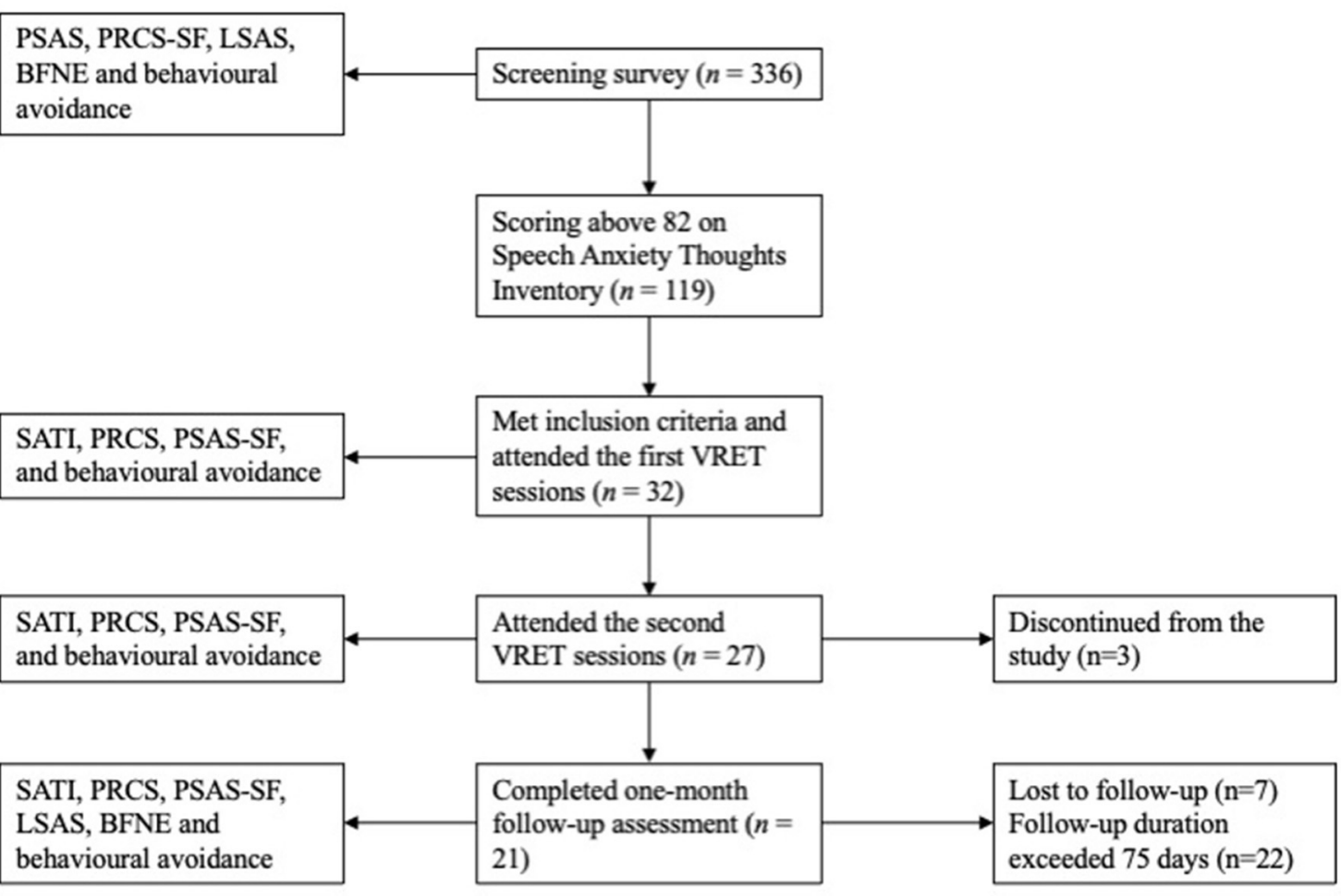

FIGURE 2 | Flow diagram of participant retention at each stage of the study; BFNE, brief fear of negative evaluation scale; LSAS, Liebowitz social anxiety scale; PRCS-SF, personal report of confidence as a speaker; PSAS-SF, Public-speaking anxiety scale-short form; SATI, speech anxiety thoughts inventory; SUDS, subjective units of distress scale.

\section{Procedure}

Invited participants completed the online screening survey on an average of 60 days (median $=46$ days, $\mathrm{SD}=59.4$ ) before they took part in Session 1 . The screening survey comprised the SATI, PSAS, PRCS-SF, LSAS, BFNE and SUDS for behavioral avoidance (Figure 2). Participants who fulfilled the selection criteria for the highest SATI scores were invited to attend the 2 weekly hour-long sessions (number of days between sessions mean = 7.8 , median $=7, \mathrm{SD}=5.3$ ). Participants were given a hard copy of the PowerPoint slides containing the speech prompts a few minutes before they wore the VR headset to familiarize themselves with the suggested speaking points. Participants were given the following instructions,

'You will have three minutes to look over the notes before we begin the virtual-reality experiment. You will see the notes in the VR environment. Don't read the notes - talk about what you want to talk about regarding your experiences. The notes are there to give you prompts when you run out of things to say. Don't worry if you go "off topic"! The aim is to keep you talking for 20 minutes, and NOT the quality of your presentation. Make it personal - give your views and opinions, and share personal stories and examples. Don't rush. Speak slowly and clearly. Spend time elaborating on the notes. You can switch to a higher level on any of the features I mentioned about whenever you enter the pause menu. You are encouraged to switch to a higher level in any of these individual areas whenever you feel comfortable.'

Participants engaged in the 20-min VRET speech in 5-min blocks, which was interspersed by four up-to-1-min intervals to allow the participant to manipulate the environment, should they choose to. Participants completed the self-report questionnaires at the end of each 20-min session and 1 month after the second session (number of days between Session 2 and follow-up mean $=56.9$, median $=45, \mathrm{SD}=42.5$ ). The Business, Law and Social Sciences College Research Ethics Committee at Nottingham Trent University approved the study (ethics application number No. 2017/82). Participants gave informed consent and were given a $£ 10$ shopping voucher for each experimental session attended and awarded research credits.

\section{Statistical Analyses}

Thirty-two participants completed Session 1, 27 completed Session 2, and 21 completed the follow-up assessment (Figure 2; note that data from two participants exceeded the 75-day followup limit and were excluded at follow-up). Participants were informed that they could withdraw without giving a reason. Final completers $(n=21)$ and non-completers $(n=11)$ did not 
TABLE 2 | Self-guided exposure to social threat within the virtual environment ${ }^{\dagger}$.

\begin{tabular}{|c|c|c|c|c|c|c|c|}
\hline & Pause 1 & Pause 2 & Pause 3 & Pause 4 & $F$-statistic (df) & $P$-value & Effect size $\left(\eta^{2}\right)$ \\
\hline \multicolumn{8}{|l|}{ Session $1(n=32)$} \\
\hline Audience size & $1.81(0.64)$ & $2.38(0.61)$ & $2.66(0.54)$ & $2.78(0.49)$ & $30.36(3,93)$ & $<0.001$ & 0.49 \\
\hline Audience reaction & $1.59(0.76)$ & $1.91(0.69)$ & $2.19(0.82)$ & $2.09(0.86)$ & $4.62(3,93)$ & 0.005 & 0.13 \\
\hline Number of prompts & $1.53(0.72)$ & $1.66(0.74)$ & $1.81(0.82)$ & $1.78(0.83)$ & $2.17(3,93)$ & 0.121 & 0.06 \\
\hline Salience of self & $1.81(0.78)$ & $2.12(0.79)$ & $2.44(0.80)$ & $2.44(0.84)$ & $12.44(3,93)$ & $<0.001$ & 0.29 \\
\hline \multicolumn{8}{|l|}{ Session $2(n=25)$} \\
\hline Audience size & $2.37(0.74)$ & $2.67(0.55)$ & $2.74(0.45)$ & $2.81(0.40)$ & $7.31(3,78)$ & 0.002 & 0.22 \\
\hline Audience reaction & $1.85(0.82)$ & $2.18(0.88)$ & $2.41(0.84)$ & $2.41(0.84)$ & $5.88(3,78)$ & 0.007 & 0.18 \\
\hline Number of prompts & $1.78(0.80)$ & $1.81(0.79)$ & $1.93(0.83)$ & $2.00(0.83)$ & $2.10(3,78)$ & 0.143 & 0.07 \\
\hline Salience of self & $2.30(0.82)$ & $2.55(0.75)$ & $2.66(0.68)$ & $2.78(0.58)$ & $6.83(3,78)$ & 0.002 & 0.21 \\
\hline
\end{tabular}

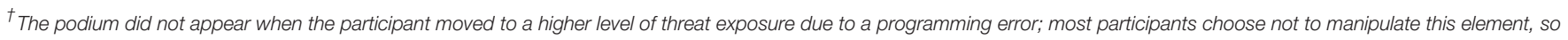
results for manipulation of distance from audience are not reported.

differ demographically or on any self-report measure at baseline or at the end of Session 1 (Table 1). Multiple imputation was used to replace the missing values of the self-report assessments and heartrate during the VRET sessions [c.f. (69)]. An iterative Markov chain Monte Carlo (MCMC) method was used to perform the multiple imputation due to the monotonic nature of the missing responses. Data on the levels of exposure to each element that participants could manipulate were missing, but not replaced due to their ordinal nature.

A separate analysis of variance (ANOVA) was performed on each VRET session with time as the independent variable (four pauses) and the four elements of graded exposure as the dependent variables (hypothesis 1). Further ANOVAs were performed with time $(\times 5$ for anxiety and arousal SUDS and $\times 6$ for heartrate) and session $(\times 2)$ as independent variables, and anxiety SUDS, arousal SUDS and heartrate as the dependent variables (hypothesis 2). An ANOVA was performed with time $(\times 3$, baseline, post-treatment and 1-month follow-up) as the independent variable and the scores on SATI, PSAS, PRCS-SF, avoidance of giving a speech, BFNE and LSASfear of performance as the dependent variables (hypothesis 3). Post hoc Bonferroni-corrected pairwise comparisons compared timepoints. The change in anxiety, relative to baseline, was calculated as: (anxiety at baseline - anxiety post Session 2 or at follow-up)/anxiety at baseline. The change, relative to baseline, in SUDS anxiety and arousal was correlated against the change, relative to baseline, in SATI, PRCS-SF, PSAS, LSAS and BFNE post-treatment and at 1-month follow-up (hypothesis 4).

\section{RESULTS}

\section{Graded Exposure to Social Threat in the Virtual Environment}

Participants chose to increase their self-guided exposure to audience size, audience reaction and salience of self by the last pause of Session 1 relative to the first pause of Session 1 (Table 2 and Figure 3A). Likewise, participants chose to increase their self-guided exposure by the last pause of Session 2 relative to the first pause of Session 2. The level of the number of speech prompts did not change significantly in either session. Participants also exhibited greater exposure to audience size, $F_{(1,26)}=43.87, p<0.001, \eta^{2}=0.63$; audience reaction, $F_{(1,26)}=10.98, p=0.003, \eta^{2}=0.30$; number of prompts, $F_{(1,26)}=4.97, p=0.035, \eta^{2}=0.16$; and salience of self, $F_{(1,26)}=26.08, p<0.001, \eta^{2}=0.50$, at the last pause of Session 2 relative to the first pause of Session 1 .

\section{Changes in Anxiety, Arousal and Heartrate During the VRET Sessions}

There was a main effect of time on SUDS-anxiety over the two sessions $F_{(4,124)}=9.24, p<0.001, \eta^{2}=0.23$ (Figure 3B). Post hoc Bonferroni-corrected pairwise comparisons revealed reduced anxiety by the end of each VRET session relative to the first two pauses, $p \leq 0.001$. There was a main effect of session on SUDSanxiety, $F_{(1,31)}=30.77, p<0.001, \eta^{2}=0.50$. SUDS-anxiety decreased by Session 2 relative to the first pause of Session 1, mean difference $=24.94, F_{(1,31)}=40.33, p<0.001, \eta^{2}=0.56$.

There was a significant main effect of session, $F_{(1,31)}=11.87$, $p=0.002, \eta^{2}=0.28$. There was no main effect of time on SUDSarousal, $F_{(4,124)}=2.60, p=0.08, \eta^{2}=0.08$. Arousal was lower at Session 2 than at session 1. SUDS-arousal decreased by Session 2 relative to baseline, mean difference $=15.99, F_{(1,31)}=10.02, p$ $=0.003, \eta^{2}=0.24$. There was a main effect of time on heartrate, $F_{(5,155)}=3.00, p=0.013, \eta^{2}=0.09$, but no main effect of session on heartrate, $F_{(1,31)}=0.30, p=0.59, \eta^{2}=0.01$ (Figure 3C). Heartrate decreased by the end of Session 2 relative to baseline, mean difference $=4.55, \mathrm{SD}=11.01, F_{(1,31)}=5.48, p=0.002, \eta^{2}$ $=0.15$.

\section{Change in Self-Reported PSA Over Time}

There was a significant main effect of time on PSA as measured by SATI, PSAS, PRCS-SF, avoidance of giving a speech (single item question), BFNE and LSAS - fear of performance (Table 3 ). Bonferroni-corrected post hoc pairwise comparisons revealed improvement at Session 1, Session 2 and 1-month followup relative to baseline, $p \leq 0.01$, on the SATI, PSAS and avoidance of giving a speech. PSAS and PRCS-SF scores 
A

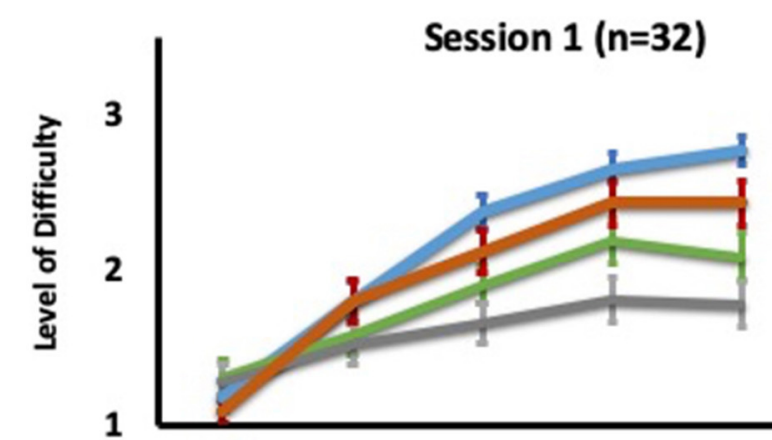

Session 2 ( $n=26)$

Baseline Pause 1 Pause 2 Pause 3 Pause 4

Baseline Pause 1 Pause 2 Pause 3 Pause 4 Audience size $\longrightarrow$ Audience reaction

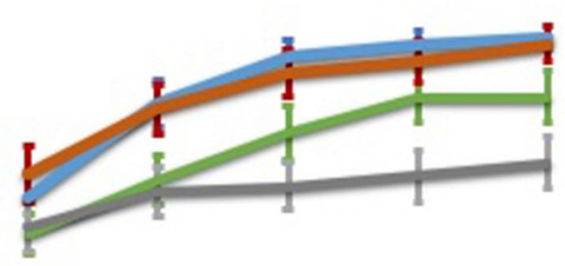

B

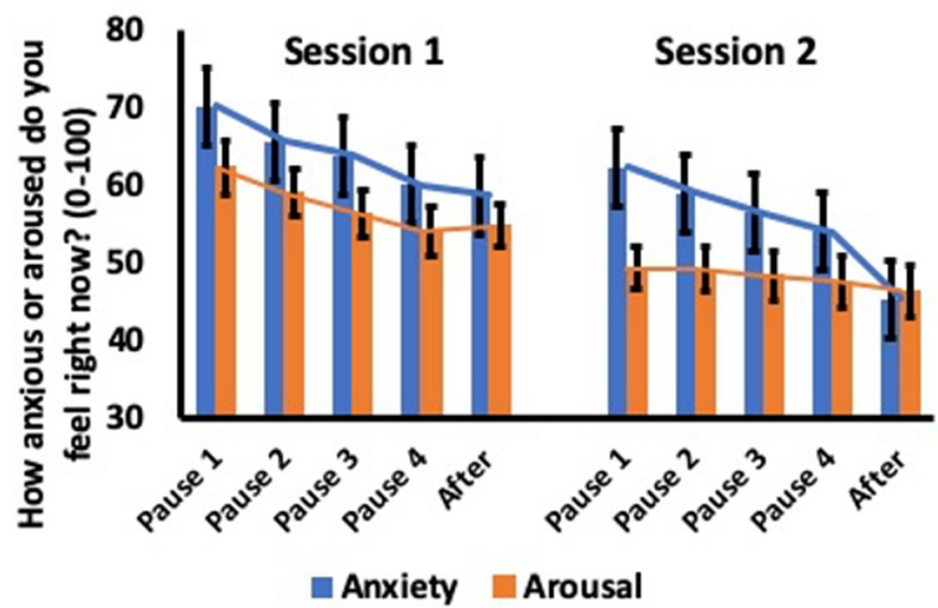

C

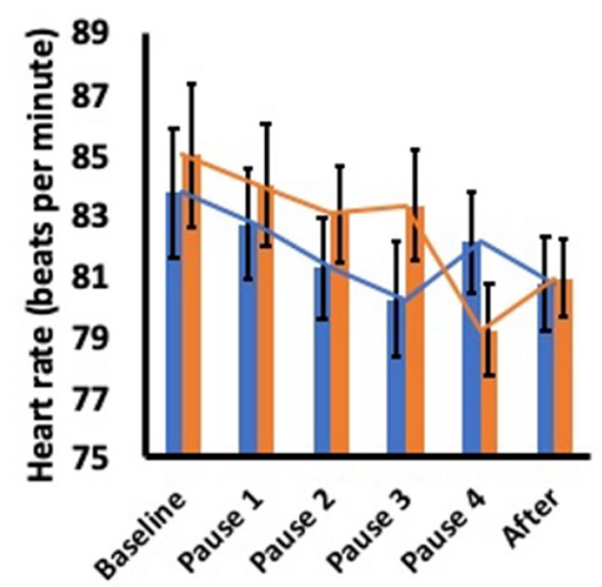

- Session 1 Session 2

FIGURE 3 | Participant changes in exposure to social threat at each 4-min pause within the virtual environment in (A) modifying the elements of the social threat, (B) anxiety and arousal and (C) heartrate.

TABLE 3 | Change in PSA from baseline, to Session 1, Session 2 and one-month follow-up.

\begin{tabular}{|c|c|c|c|c|c|c|c|c|}
\hline Measure & Baseline (A) & Session 1 (B) & Session 2 (C) & $\begin{array}{c}\text { 1-month } \\
\text { follow-up (D) }\end{array}$ & $F(d f)$ & $P$-value & $\begin{array}{c}\text { Effect } \\
\text { size }\left(\eta^{2}\right)\end{array}$ & Pairwise comparisons \\
\hline SATI & $96.65(7.77)$ & $84.81(19.00)$ & $78.84(20.06)$ & $71.18(17.99)$ & $21.80(3,93)$ & $<0.001$ & 0.41 & $A>B^{\star}, A>C$ and $D^{\star \star \star}, B>D^{\star \star}, C>D^{\star}$ \\
\hline PRCS-SF & $1.17(0.14)$ & $1.28(0.21)$ & $2.15(0.24)$ & $1.36(0.22)$ & $214.1(3,93)$ & $<0.001$ & 0.87 & $\mathrm{~A}<\mathrm{C}$ and $\mathrm{D}^{\star \star \star}, \mathrm{B}<\mathrm{C}^{\star \star \star}, \mathrm{D}<\mathrm{C}^{\star \star \star}$ \\
\hline Speech avoidance & $89.69(19.10)$ & $55.62(21.99)$ & 53.77 (23.59) & $47.48(20.82)$ & $38.19(3,93)$ & $<0.001$ & 0.55 & $\mathrm{~A}>\mathrm{B}, \mathrm{C}$ and $\mathrm{D}^{\star \star \star}$ \\
\hline BFNE & $49.68(9.73)$ & - & $46.70(9.59)$ & $43.10(9.31)$ & $8.93(2,62)$ & 0.002 & 0.22 & $A>D^{* *}, C>D^{*}$ \\
\hline LSAS-P-avoid & $17.91(6.60)$ & - & $17.31(6.22)$ & $15.82(5.49)$ & $2.03(2,62)$ & 0.140 & 0.06 & \\
\hline LSAS-S-anx & $18.69(7.66)$ & - & $17.69(6.66)$ & $16.44(7.01)$ & $2.48(2,62)$ & 0.092 & 0.07 & \\
\hline LSAS-S-avoid & $16.62(7.52)$ & - & $16.17(6.39)$ & $14.59(6.18)$ & $1.64(2,62)$ & 0.203 & 0.05 & \\
\hline
\end{tabular}

${ }^{*} p<0.05 ;{ }^{* *} p<0.01 ;{ }^{* \star *} p<0.001$; BFNE, brief fear of negative evaluation; LSAS, Liebowitz social anxiety scale; LSAS subscales: P-anx, performance anxiety; P-avoid, performance avoidance; S-anx, social anxiety; S-avoid, social avoidance; PRCS-SF, personal report of confidence as a speaker-short form; PSAS, public speaking anxiety scale; SATI, social anxiety thoughts inventory.

improved at Session 2 relative to Session $1, p \leq 0.01$. BFNE and LSAS-fear of performance scores improved at follow-up relative to baseline and Session $2, p<0.02$. Only the SATI score improved at follow-up relative to both Sessions 1 and $2, p<0.03$. PRCS-SF scores declined at follow-up relative to Session $2, p<0.001$. 


\section{Correlation Between Change in Anxiety and Arousal During VRET Sessions With Change in PSA}

Improvement in SUDS-anxiety from the first pause of Session 1 to post-Session 2 correlated with (1) improvement in PSAS pretherapy to post-Session $2, r=0.40, p=0.023$, (2) improvement in BFNE 2 pre-therapy to post-Session 2, $r=0.40, p=0.022$, and (3) improvement in BFNE pre-therapy to follow-up, $r=0.44, p$ $=0.012$.

\section{DISCUSSION}

This is the first study to systematically examine the feasibility of self-guided VRET for PSA. This self-guided VRET aims to encourage individuals with high self-reported PSA to voluntarily pace their gradual exposure to virtual social threat (hypothesis 1). These findings support the hypotheses that reductions in self-reported anxiety and physiological arousal can accompany the ongoing self-guided desensitization to virtual social threat (hypothesis 2). Furthermore, self-guided VRET improves PSA after intervention and at 1-month follow-up (hypothesis 3). Finally, a reduction in anxiety during the VRET sessions relates to an overall improvement in PSA after the intervention and 1 month later (hypothesis 4). These findings are discussed further.

On average, participants increased their exposure to all four available elements of social threat over the course of the two VRET sessions. Within each session, participants (on average) increased their graded exposure to three out of the four elements of social threat, namely audience size, audience reaction and salience of self, and participants made full use of the range of exposures offered. This preliminary evidence suggests that self-guided exposure has the potential to desensitize individuals with high PSA to social threat without risking exposure to excessive fear. The possible health beliefs that accompany this improvement could be that participants gain a sense of control over one's health and feel empowered and motivated to engage with treatment $(34,35)$. Future studies could explicitly test the role of health beliefs when engaging in self-guided VRET.

Alongside this increased exposure to virtual social threat, the self-guided VRET produced reductions in anxiety during the VRET sessions, improved subjective and physiological levels of arousal (heartrate). Additionally, participants showed overall improvement in PSA across the two sessions. These findings suggest that self-pacing one's exposure to virtual social threat could reliably alleviate anxiety and arousal when using the application. In addition, the VRET-linked reduction in anxiety found during the VRET sessions related to an overall improvement in PSA after the two sessions and to a further improvement in fear of negative evaluation 1 month later. Hence, these improvements could be linked to long-term improvement in fear of negative evaluation. Exposure to social threats within the virtual environment could mean reduced perceived social anxiety in real life, such as being concerned about social judgment. Less anxiety within the virtual environment does translate to less anxiety in real life, since VRET reduces reallife self-reported anxiety and length of speech during a speech in front of an audience $(24,25)$. The self-paced exposure to virtual social threat could encourage effortful emotion regulation (70). The relief in anxiety during application could modify cognitive elements of PSA, such as reevaluation of irrational beliefs, anticipated anxious rumination and self-referential bias $(71,72)$. Following the intervention, a participant informed the research team: 'I did a presentation last week. While I was still anxious and I found my heart pounded, I definitely noticed a difference! I didn't stutter and I was able to look my audience in the eyes. I'm definitely still anxious with presentations, but it's made me more able to face them.' Again, future investigations should examine such mechanisms of emotion regulation and perceived control that aid improvement in fear of negative evaluation.

The maintenance of the improvement in PSA 1 month later could suggest that self-guided VRET addresses the core features of PSA, namely fear of negative evaluation and fear of performance. Fear of negative evaluation is a key feature of social anxiety. It is characterized by a strong negative self-referential bias and irrational thoughts, such as worrying about how others feel about you and perceiving criticism and rejection from others (73). The self-guided VRET may help clients to challenge their beliefs and biases toward the virtual social threats, such as virtual audience members shaking their heads, and to transfer these skills to real life. Virtual exposure to threat-provoking situations, including public-speaking, translates to "real life" threat (22). This improvement in fear of negative evaluation following VRET is consistent with the findings of Anderson et al.'s (24) study, but not Kampmann et al.'s (16) study. Participants who received therapist-led VRET and performed homework assignments alongside the VRET showed an improvement in fear of negative evaluation (24). Participants who did not perform homework assignments did not show this improvement (16). The self-guided VRET might challenge perceptions of social threat in real life. Setting homework assignments for socially anxious individuals to practice these skills could have added long-term value following self-guided VRET. Future investigations should determine how long the improvement in PSA is sustained. For example, it is known that a single session of self-guided VRET for fear of spiders can sustain reduced anxiety for up to 12 months post-treatment (43), and self-guided VRET for SAD may offer similar effects.

\section{The Psychophysiological Mechanisms of Responsiveness to Self-Guided VRET}

Physiological habituation happens when adapting to stress. High social anxiety can delay this habituation (74). The current study found a reduction in heartrate of 4.5 beats per minute by the end of VRET Session 2 relative to baseline, and this reduction equated to large effect size. This reduction in heartrate suggests habituation to delivering a speech to the virtual audience. A virtual exposure to social threat over a 4-week period as part of a therapist-guided VRET for PSA has previously shown to reduce heartrate (55). In contrast, other research has shown that brief, 3-minute, exposure to virtual social threat does not change heartrate when the virtual audience gradually increases its display of 
threat (23). Thus, the duration of exposure to social threat may determine the amount of physiological habituation.

\section{Limitations, Technological Advances to Enhance the VRET Experience and Therapeutic Implications}

This study was a feasibility study. It did not include a control intervention, such as a virtual-reality-guided breathing exercise, and so it did not determine whether a routine 20-min exercise would produce a similar improvement in PSA, as participants naturally regress to the mean. A full randomized-controlled trial must test whether multiple sessions of the intervention are beneficial and how the intervention translates to real life, such as delivering a speech in vivo. Participants predominantly had a subclinical level of PSA; so, the findings may not generalize to clinical SAD. Furthermore, therapeutic effects could be confounded by participant preference effects that are specific to the current self-guided VRET, namely the size and reaction of the audience, the number of speech prompts and the topic of the speech, and those that are general to intervention, such as autonomy (75) and attitude to intervention (76).

The manipulation of certain elements in the current VRET was successful in reducing anxiety. Going forward, machine learning could be used to identify the best candidate indicators of arousal, such as galvanic skin response (GSR), pupil diameter, heart rate (HR), and electromyography (77). Offering participants biofeedback about such arousal from heartrate and electroencephalography could enhance response to exposure therapy for SAD (78). Most studies (65\%) offering biofeedback as an intervention for psychiatric disorders report symptom improvement (79), including control over threatening thoughts (80). Artificial intelligence could study the participant's voice stress patterns (29) and physiological arousal from virtual social threat and automatically up- or downgrade exposure to virtual threat (29). Further elements could also be added to enhance the realism of the virtual threat, for example, allowing avatars in the virtual audience to offer verbal auditory feedback (81) and allowing avatars to make natural small and gross movements, such as leaving the room or muttering to a neighbour (82).

\section{REFERENCES}

1. Stein MB, Stein DJ. Social anxiety disorder. Lancet. (2008) 371:1115-25. doi: 10.1016/S0140-6736(08)60488-2

2. Stein DJ, Lim CCW, Roest AM, de Jonge P, Aguilar-Gaxiola S, Al-Hamzawi A, et al. The cross-national epidemiology of social anxiety disorder: data from the World Mental Health Survey Initiative. BMC Med. (2017) 15:143. doi: 10.1186/s12916-017-0889-2

3. Kessler RC, Berglund P, Demler O, Jin R, Walters EE. Lifetime prevalence and age-of-onset distributions of DSM-IV disorders in the National Comorbidity Survey Replication. Arch General Psychiatry. (2005) 62:593-602. doi: 10.1001/archpsyc.62.6.593

4. Van Ameringen M, Mancini C, Farvolden P. The impact of anxiety disorders on educational achievement. J Anxiety Disord. (2003) 17:561-71. doi: 10.1016/S0887-6185(02)00228-1
This study is preliminary evidence of the feasibility of selfguided VRET. Self-guided VRET enables people with high PSA to voluntarily increase their exposure to virtual social threat, reduce short-term anxiety and physiological arousal, and improve perceived PSA up to a month after intervention. Such self-guided exposure could reduce the fear of negative evaluation, that is a core feature of social anxiety, and help people with high PSA to see the social threat objectively. Self-guided VRET has the potential to enhance engagement with services and augment treatment effects before, during and after treatment (31).

\section{DATA AVAILABILITY STATEMENT}

The datasets presented in this study can be found in online repositories. The names of the repository/repositories and accession number(s) can be found at: https://osf.io/tje8g.

\section{ETHICS STATEMENT}

The studies involving human participants were reviewed and approved by Business, Law, and Social Sciences College Research Ethics Committee at Nottingham Trent University. The participants provided their written informed consent to participate in this study.

\section{AUTHOR CONTRIBUTIONS}

EZ, PP, NH, DB, and AS designed the study and VRET. SB developed the virtual interface of the virtual-reality exposure therapy. $\mathrm{BH}$ and $\mathrm{RD}$ conducted the data collection. PP, NH, EZ, $\mathrm{BH}$, and $\mathrm{RD}$ performed the data analysis. PP wrote the first draft of the paper. All authors contributed to the interpretation of the results, writing and editing of the manuscript, and approved the final version of the manuscript.

\section{FUNDING}

Funding for this study was provided by the Higher Education Funding Council for England quality-related research (QR) funding awarded to the University.

5. Wittchen HU, Fuetsch M, Sonntag H, Müller N, Liebowitz M. Disability and quality of life in pure and comorbid social phobia. Findings from a controlled study. Eur Psychiatry. (2000) 15:46-58. doi: 10.1016/S0924-9338(00) 00211-X

6. Katzelnick DJ, Greist JH. Social anxiety disorder: an unrecognized problem in primary care. J Clin Psychiatry. (2001) 62(Suppl 1):11-6.

7. Grant BF, Hasin DS, Blanco C, Stinson FS, Chou S, Goldstein RB, et al. The epidemiology of social anxiety disorder in the United States: results from the national epidemiologic survey on alcohol and related conditions. J Clin Psychiatry. (2005) 66:1351-61. doi: 10.4088/JCP.v66n1102

8. Chartier-Otis M, Perreault M, C. Be langer, Determinants of barriers to treatment for anxiety disorders. Psychiatr Quart. (2010) 81:127-38. doi: 10.1007/s11126-010-9123-5

9. Kim EH, Hong Y-J, Kim M-K, Jung YH, Kyeong S, Kim J-J. Effectiveness of self-training using the mobile-based virtual reality program in patients 
with social anxiety disorder. Comput Hum Behav. (2017) 73:614-9. doi: 10.1016/j.chb.2017.04.017

10. Kaplan S, Swee M, Heimberg R. Psychological Treatments for Social Anxiety Disorder. Oxford Research Encyclopedia of Psychology. Available online at: https://oxfordre.com/psychology/view/10.1093/acrefore/9780190236557. 001.0001/acrefore-9780190236557-e-98 (accessed June 8, 2021).

11. NICE. Social Anxiety Disorder: Recognition, Assessment and Treatment. Leicester: British Psychological Society (2013).

12. Barkowski S, Schwartze D, Strauss B, Burlingame GM, Barth J, Rosendahl J. Efficacy of group psychotherapy for social anxiety disorder: a metaanalysis of randomized-controlled trials. J Anxiety Disord. (2016) 39:44-64. doi: 10.1016/j.janxdis.2016.02.005

13. Carl E, Stein AT, Levihn-Coon A, Pogue JR, Rothbaum B, Emmelkamp P, et al. Virtual reality exposure therapy for anxiety and related disorders: a metaanalysis of randomized controlled trials. J Anxiety Disord. (2019) 61:27-36. doi: 10.1016/j.janxdis.2018.08.003

14. Valmaggia LR, Latif L, Kempton MJ, Rus-Calafell M. Virtual reality in the psychological treatment for mental health problems: a systematic review of recent evidence. Psychiatry Res. (2016) 236:189-95. doi: 10.1016/j.psychres.2016.01.015

15. Horigome T, Kurokawa S, Sawada K, Kudo S, Shiga K, Mimura M, et al. Virtual reality exposure therapy for social anxiety disorder: a systematic review and meta-analysis. Psychol Med. (2020) 50:2487-97. doi: 10.1017/S0033291720003785

16. Kampmann IL, Emmelkamp PMG, Hartanto D, Brinkman WP, Zijlstra BJH, Morina N. Exposure to virtual social interactions in the treatment of social anxiety disorder: a randomized controlled trial. Behav Res Ther. (2016) 77:14756. doi: 10.1016/j.brat.2015.12.016

17. Botella C, Fernández-Álvarez J, Guillén V, García-Palacio A, Baños R. Recent progress in virtual reality exposure therapy for phobias: a systematic review. Curr Psychiatry Rep. (2017) 19:42. doi: 10.1007/s11920-017-0788-4

18. Safir MP, Wallach HS, Bar-Zvi M. Virtual reality cognitive-behavior therapy for public speaking anxiety: one-year follow-up. Behav Modif. (2012) 36:23546. doi: 10.1177/0145445511429999

19. Beidel DC, Turner SM. Shy Children, Phobic Adults: Nature and Treatment of Social Anxiety Disorder. 2nd ed. Washington, DC: American Psychological Association (2007).

20. Ruscio A, Brown T, Chiu W, Sareen J, Stein M, Kessler R. Social fears and social phobia in the USA: results from the National Comorbidity Survey Replication. Psychol Med. (2008) 38:15-28. doi: 10.1017/S0033291707001699

21. Fumark T, Tilfors M, Everz PO. Social phobia in the general population: prevalence and sociodemographic profile. Soc Psychiatry Psychiatr Epidemiol. (1999) 38:416-24. doi: 10.1007/s001270050163

22. Owens ME, Beidel DC. Can virtual reality effectively elicit distress associated with social anxiety disorder? J Psychopathol Behav Assess. (2015) 37:296-305. doi: 10.1007/s10862-014-9454-x

23. Kishimoto $T$, Ding $X$. The influences of virtual social feedback on social anxiety disorders. Behav Cogn Psychother. (2019) 47:726-35. doi: 10.1017/S1352465819000377

24. Anderson PL, Price M, Edwards SM, Obasaju MA, Schmertz SK, Zimand E, et al. Virtual reality exposure therapy for social anxiety disorder: a randomized controlled trial. J Consult Clin Psychol. (2013) 81:751-60. doi: 10.1037/a0033559

25. Wallach HS, Safir MP, Bar-Zvi M. Virtual reality cognitive behavior therapy for public speaking anxiety: a randomized clinical trial. Behav Modif. (2009) 33:314-38. doi: 10.1177/0145445509331926

26. Pertaub DP, Slater M, Barker C. An experiment on public speaking anxiety in response to three different types of virtual audience. Presence Teleoper Virtual Environ. (2002) 11:68-78. doi: 10.1162/105474602317343668

27. Powers MB, Briceno NF, Gresham R, Jouriles EN, Emmelkamp PMG, Smits JAJ, et al. Do conversations with virtual avatars increase feelings of social anxiety? J Anxiety Disord. (2013) 27:398-403. doi: $10.1016 /$ j.janxdis.2013.03.003

28. Anderson PL, Anderson SM, Goodnight JR. Virtual reality and exposure group therapy for social anxiety disorder: results from a 4-6 year follow-up. Cogn Ther Res. (2017) 41:230-6. doi: 10.1007/s10608-016-9820-y

29. Ben-Moussa M, Rubo M, Debracque C, Lange WG. DJInnI: a novel technology supported exposure therapy paradigm for SAD combining virtual reality and augmented reality. Front Psychiatry. (2017) 8:26. doi: 10.3389/fpsyt.2017.00026

30. Lindner P. Better, virtually: the past, present, and future of virtual reality cognitive behavior therapy. Int J Cogn Ther. (2020) 14:23-46. doi: 10.1007/s41811-020-00090-7

31. Lindner P, Miloff A, Fagernäs S, Andersen J, Sigeman M, Andersson G, et al. Therapist-led and self-led one-session virtual reality exposure therapy for public speaking anxiety with consumer hardware and software: a randomized controlled trial. J Anxiety Disord. (2019) 61:45-54. doi: 10.1016/j.janxdis.2018.07.003

32. Donker T, Cornelisz I, van Klaveren C, van Straten A, Carlbring P, Cuijpers P, et al. Effectiveness of self-guided app-based virtual reality cognitive behavior therapy for acrophobia: a randomized clinical trial. JAMA Psychiatry. (2019) 76:682-90. doi: 10.1001/jamapsychiatry.2019.0219

33. Freeman D, Haselton P, Freeman J, Spanlang B, Kishore S, Albery E, et al. Automated psychological therapy using immersive virtual reality for treatment of fear of heights: a single-blind, parallel-group, randomised controlled trial. Lancet Psychiatry. (2018) 5:625-32. doi: 10.1016/S2215-0366(18)30226-8

34. Sirur R, Richardson J, Wishart L, Hanna S. The role of theory in increasing adherence to prescribed practice. Physiother Can. (2009) 61:68-77. doi: 10.3138/physio.61.2.68

35. Alami S, Stieglitz J, Kaplan H, Gurven M. Low perceived control over health is associated with lower treatment uptake in a high mortality population of Bolivian forager-farmers. Soc Sci Med. (2018) 200:156-65. doi: 10.1016/j.socscimed.2018.01.017

36. Bhanji J, Kim E, Delgado M. Perceived control alters the effect of acute stress on persistence. J Exp Psychol. (2016) 145:356-65. doi: 10.1037/xge0000137

37. Evans B, Fischer D. A hierarchical model of participatory decision-making, job autonomy, and perceived control. Hum Relat. (1992) 45:1169-89. doi: $10.1177 / 001872679204501103$

38. Fishman EJ, Husman J. Extending attribution theory: considering students' perceived control of the attribution process. J Educ Psychol. (2017) 109:559-73. doi: 10.1037/edu0000158

39. Buchholz JL, Abramowitz JS. The therapeutic alliance in exposure therapy for anxiety-related disorders: a T critical review. J Anxiety Disord. (2020) 70:102194. doi: 10.1016/j.janxdis.2020.102194

40. Healey A, Mansell W, Tai S. An experimental test of the role of control in spider fear. J Anxiety Disord. (2017) 49:12-20. doi: 10.1016/j.janxdis.2017.03.005

41. Mansell W, Huddy V. Why do we need computational models of psychological change and recovery, and how should they be designed and tested? Front Psychiatry. (2020) 11:624. doi: 10.3389/fpsyt.2020. 00624

42. Motraghi TE, Seim RW, Meyer EC, Morissette S. Virtual reality exposure therapy for the treatment of posttraumatic stress disorder: a methodological review using CONSORT guidelines. J Clin Psychol. (2014) 70:197-208. doi: $10.1002 /$ jclp. 22051

43. Miloff A, Lindner P, Dafgård P, Deak S, Garke M, Hamilton W, et al. Automated virtual reality exposure therapy for spider phobia vs. in-vivo onesession treatment: a randomized non-inferiority trial. Behav Res Ther. (2019) 118:130-40. doi: 10.1016/j.brat.2019.04.004

44. Poeschl S. Virtual reality training for public speaking-a QUesT-Vr Framework validation. Front ICT. (2017) 4:13. doi: 10.3389/fict.2017.00013

45. Aymerich-Franch L, Kizilcec RF, Bailenson JN. The relationship between virtual self similarity and social anxiety. Front Hum Neurosci. (2014) 8:944. doi: 10.3389/fnhum.2014.00944

46. Fergus TA, Valentiner DP, McGrath PB, Stephenson K, Gier S, Jecius S. The fear of positive evaluation scale: psychometric properties in a clinical sample. J Anxiety Disord. (2009) 23:1177-83. doi: 10.1016/j.janxdis.2009.07.024

47. Rapee RM, Heimberg RG. A cognitive-behavioral model of anxiety in social phobia. Behav Res Ther. (1997) 35:741-56. doi: 10.1016/S0005-7967(97)00022-3

48. Hirsch CR, Clark DM. Information-processing bias in social phobia. Clin Psychol Rev. (2004) 24:799-825. doi: 10.1016/j.cpr.2004.07.005

49. Daly JA, Vangelisti AL, Lawrence SG. Self-focused attention and public speaking anxiety. Pers Individ Dif. (1989) 10:903-13. doi: 10.1016/0191-8869(89)90025-1 
50. Brown MA, Stopa L. The spotlight effect and the illusion of transparency in social anxiety. J Anxiety Disord. (2007) 21:804-19. doi: 10.1016/j.janxdis.2006.11.006

51. Slater M. Presence and emotions. CyberPsychol Behav. (2004) 7:121. doi: $10.1089 / 109493104322820200$

52. Maples-Keller JL, Bunnell BE, Kim SJ, Rothbaum BO. The use of virtual reality technology in the treatment of anxiety and other psychiatric disorders. Harvard Rev Psychiatry. (2017) 25:103-13. doi: 10.1097/HRP.0000000000000138

53. Morina N, Brinkman W-P, Hartanto D, Emmelkamp PMG. Sense of presence and anxiety during virtual social interactions between a human and virtual humans. PeerJ. (2014) 2:e337. doi: 10.7717/peerj.337

54. Pujol J, Giménez M, Oritz H, Soriano-Mas C, López-Solà M, Farré M, et al. Neural response to the observable self in social anxiety disorder. PsycholMed. (2013) 43:721-31. doi: 10.1017/S0033291712001857

55. Harris SR, Kemmerling RL, North MM. Brief virtual reality therapy for public speaking anxiety. Cyberpsychol Behav. (2002) 5:543-50. doi: $10.1089 / 109493102321018187$

56. Cho Y, Smits JAJ, Telch MJ. The speech anxiety thoughts inventory: scale development and preliminary psychometric data. Behav Res Ther. (2004) 42:13-25. doi: 10.1016/S0005-7967(03)00067-6

57. Bartholomay EM, Houlihan DD. Public speaking anxiety scale: preliminary psychometric data and scale validation. Pers Individ Dif. (2016) 94:211-5. doi: 10.1016/j.paid.2016.01.026

58. Hook JN, Smith CA, Valentiner DP. A short-form of the personal report of confidence as a speaker. Pers Individ Dif. (2008) 44:1306-13. doi: 10.1016/j.paid.2007.11.021

59. Martinez-Pecino R, Durán M. Social communication fears: factor analysis and gender invariance of the short-form of the personal report of confidence as a speaker in Spain. Pers Individ Dif. (2013) 55:680-4. doi: 10.1016/j.paid.2013.05.017

60. Liebowitz MR. Social phobia. Mod Probl Pharmacopsychiatry. (1987) 22:14173. doi: $10.1159 / 000414022$

61. Heimberg RG, Horner KJ, Juster HR, Safren SA, Brown EJ, Schneier FR, et al. Psychometric properties of the Liebowitz social anxiety scale. Psychol Med. (1999) 29:199-212. doi: 10.1017/S0033291798007879

62. Carleton RN, McCreary DR, Norton PJ, Asmundson GJG. Brief fear of negative evaluation scale-revised. Depress Anxiety. (2006) 23:297-303. doi: $10.1002 /$ da. 20142

63. Wolpe J. Psychotherapy by Reciprocal Inhibition. Stanford, CA: Stanford University Press (1958).

64. Levy HC, Radomsky AS. Are all safety behaviours created equal? A comparison of novel and routinely used safety behaviours in obsessive-compulsive disorder. Cogn Behav Ther. (2016) 45:367-79. doi: 10.1080/16506073.2016. 1184712

65. Reips U, Funke F. Interval-level measurement with visual analogue scales in Internet-based research: VAS generator. Behav Res Methods. (2008) 40:699704. doi: 10.3758/BRM.40.3.699

66. Lovibond SH, Lovibond PF. Manual for the Depression, Anxiety and Stress Scales. 2nd edn. Sydney: Psychology Foundation (1995).

67. King M, Burrows G, Stanley G. Measurement of stress and arousal: Validation of the stress/arousal adjective checklist. Br J Psychol. (1983) 74:473-79. doi: 10.1111/j.2044-8295.1983.tb01880.x

68. Unity Technologies, Unity (2017). Available online at: https://unity3d.com/ (accessed June 8, 2021).

69. Del Re AC, Maisel NC, Blodgett JC, Finney JW. Intention-to-treat analyses and missing data approaches in pharmacotherapy trials for alcohol use disorders. BMJ Open. (2013) 3:e003464. doi: 10.1136/bmjopen-2013003464

70. Azbel-Jackson L, Butler LT, Ellis JA, van Reekum MC. Stay calm! Regulating emotional responses by implementation intentions: assessing the impact on physiological and subjective arousal. Cogn Emotion. (2016) 30:1107-21. doi: 10.1080/02699931.2015. 1049515
71. Booth-Butterfield M, Booth-Butterfield S. The mediating role of cognition in the experience of state anxiety. South Commun J. (1990) 56:35-4. doi: 10.1080/10417949009372814

72. Vîslă A, Cristea IA, Szentágotai TA, David D. Core beliefs, automatic thoughts and response expectancies in predicting public speaking anxiety. Pers Individ Dif. (2013) 55:856-9. doi: 10.1016/j.paid.2013. 06.003

73. Bautista C, Hope D. Fear of negative evaluation, social anxiety and response to positive and negative online social cues. Cogn Ther Res. (2015) 39:658-68. doi: 10.1007/s10608-015-9687-3

74. Sawyer CR, Behnke RR. Reduction in public speaking state anxiety during performance as a function of sensitization processes. Commun Q. (2002) 50:110-21. doi: 10.1080/014633702093 85649

75. Horan KA, Participant preference in interventions in occupational health psychology: potential implications for autonomy, (2018). Available online at: https://etd.ohiolink.edu/apexprod/rws_etd/send_file/send? accession=bgsu1524949525954918\&disposition=inline (accessed June 8, 2021).

76. Swift JK, Callahan JL. The impact of client treatment preferences on outcome: a meta-analysis. J Clin Psychol. (2009) 65:368-81. doi: 10.1002/jclp.20553

77. Erkuş EC, Purutçuoğlu V, Ari F, Gökçay D. Comparison of several machine learning classifiers for arousal classification: a preliminary study. In: 2020 Medical Technologies Congress (TIPTEKNO). IEEE (2020). p. 1-7.

78. Lin XB, Lee TS, Cheung YB, Ling J, Poon SH, Lim L, et al. Exposure therapy with personalized real-time arousal detection and feedback to alleviate social anxiety symptoms in an analogue adult sample: pilot proofof-concept randomized controlled trial. JMIR Ment Health. (2019) 6:e13869. doi: $10.2196 / 13869$

79. Schoenberg P, David A. Biofeedback for psychiatric disorders: a systematic review. Appl Psychophysiol Biofeedback. (2014) 39:109-35. doi: 10.1007/s10484-014-9246-9

80. Clamor A, Koenig J, Thayer JF, Lincoln TM. A randomizedcontrolled trial of heart rate variability biofeedback for psychotic symptoms. Behav Res Ther. (2016) 87:207-15. doi: 10.1016/j.brat.2016. 10.003

81. Nazligul MD, Yilmaz M, Gulec U, Gozcu MA, O'Connor RV, Clarke PM. Overcoming public speaking anxiety of software engineers using virtual reality exposure therapy. In: Systems, Software and Services Process Improvement. EuroSPI (2017). Communications in Computer and Information Science. vol. 748. Cham: Springer (2017). pp. 191-202.

82. Söyler E, Gunaratne C, Akbaş MI. Towards a Comprehensive Simulator for Public Speaking Anxiety Treatment, in Advances in Applied Digital Human Modeling and Simulation. Advances in Intelligent Systems and Computing. vol. 481. Cham: Springer (2017). p. 195-205. doi: 10.1007/978-3-319-416 27-4_18

Conflict of Interest: The authors declare that the research was conducted in the absence of any commercial or financial relationships that could be construed as a potential conflict of interest.

Publisher's Note: All claims expressed in this article are solely those of the authors and do not necessarily represent those of their affiliated organizations, or those of the publisher, the editors and the reviewers. Any product that may be evaluated in this article, or claim that may be made by its manufacturer, is not guaranteed or endorsed by the publisher.

Copyright $(2021$ Premkumar, Heym, Brown, Battersby, Sumich, Huntington, Daly and Zysk. This is an open-access article distributed under the terms of the Creative Commons Attribution License (CC BY). The use, distribution or reproduction in other forums is permitted, provided the original author(s) and the copyright owner(s) are credited and that the original publication in this journal is cited, in accordance with accepted academic practice. No use, distribution or reproduction is permitted which does not comply with these terms. 